\title{
Nonlinear waves in a dispersive vacuum described with a high order derivative electromagnetic Lagrangian
}

\author{
Francesco Pegoraro $\oplus^{1,2}$ and Sergei V. Bulanov $\circledast^{3,4}$ \\ ${ }^{1}$ Enrico Fermi Department of Physics, University of Pisa, 56127 Pisa, Italy \\ ${ }^{2}$ National Research Council, National Institute of Optics, via G. Moruzzi 1, 56124 Pisa, Italy \\ ${ }^{3}$ Institute of Physics of the ASCR, ELI-Beamlines project, Na Slovance 2, 18221 Prague, Czech Republic \\ ${ }^{4}$ National Institutes for Quantum and Radiological Science and Technology (QST), Kansai Photon Science \\ Institute, 8-1-7 Umemidai, Kizugawa, Kyoto 619-0215, Japan
}

(Received 13 March 2021; accepted 8 April 2021; published 14 May 2021)

\begin{abstract}
In this article we use an electromagnetic Lagrangian constructed so as to include dispersive effects in the description of an electromagnetic wave propagating in the quantum electrodynamic vacuum. This Lagrangian is Lorentz invariant, includes contributions up to six powers in the electromagnetic fields, and involves both fields and their first derivatives. Conceptual limitations inherent to the use of this higher derivative Lagrangian approach are discussed. We consider the one-dimensional spatial limit and obtain an exact solution of the nonlinear wave equation recovering the Korteveg-de Vries type periodic waves and solitons given in S. V. Bulanov et al. [Phys. Rev. D 101, 016016 (2020)].
\end{abstract}

DOI: 10.1103/PhysRevD.103.096012

\section{INTRODUCTION: WAVE EQUATIONS IN NONLINEAR QUANTUM ELECTRODYNAMICS}

Field induced polarization and birefringence of the vacuum, see e.g., Refs. [1,2], are fundamental effects predicted by quantum electrodynamics (QED). These effects arise from the process of scattering of light by light: while in classical electrodynamics electromagnetic waves do not interact in vacuum, in QED photon-photon scattering can take place in vacuum via the generation of virtual electron-positron pairs that gives rise to polarization and magnetization currents that make the vacuum respond as a material medium. The study of the nonlinear QED vacuum properties has been conducted for about a century [3-11].

Recently, interest in these effects has been rekindled by the availability of high power lasers (see review articles $[12,13])$ leading to the formulation of the plans aimed at reaching experimentally the parameters that can enable the study the nonlinear QED vacuum [14-16]. This, in turn, has motivated an intensive theoretical research program aimed at the study of the scattering of a laser pulse by a laser pulse [17-28].

The field induced vacuum polarization and birefringence can be accounted for within the framework of a local

Published by the American Physical Society under the terms of the Creative Commons Attribution 4.0 International license. Further distribution of this work must maintain attribution to the author(s) and the published article's title, journal citation, and DOI. Funded by SCOAP. approximation using the well-known Heisenberg-Euler Lagrangian in the electromagnetic action functional $[3,5]$. This approximation leads to nonlinear wave equations for the fields amplitude in vacuum that are not dispersive, i.e., that are homogeneous in the second order derivatives of the field four-vector potentials. In other words the Lagrangian does not include second order derivatives (or higher order derivatives) of the electromagnetic fields. This local approximation is valid in the long wavelength, low frequency limit, essentially requiring that the electromagnetic fields are slowly varying on the Compton scattering wavelength $\chi_{C}=\hbar / m_{e} c$, where $\hbar$ is the reduced Planck constant, $e$ and $m_{e}$ are the electron electric charge and mass, and $c$ is the speed of light in vacuum.

Nonlocal effects on the Compton scattering have been studied e.g., in Ref. [29] and, for vacuum birefringence, in Ref. [30].

For shorter wavelengths the vacuum acquires dispersion properties. In the small field amplitude limit and in the socalled cross-field approximation, these dispersive properties have been included in the "invariant photon mass" introduced in Ref. [31] (see also Refs. [32-36]). The crossfield approximation consists in approximating the interaction between a higher frequency pulse and a lower frequency pulse by taking the latter to be described by uniform and stationary electric and magnetic fields of the same amplitude and orthogonal to each other. Discussions of the QED processes beyond the constant field approximations can be found in Refs. [37-40]. The invariant photon mass refers to the higher frequency pulse; it 
depends on the relative polarization between the fields of the two pulses and can be expressed [31] in terms of the socalled quantum nonlinearity parameter $\chi_{\gamma}$. This Lorentz invariant parameter can be written for a photon impinging on an slowly varying external field as [21,31]

$$
\chi_{\gamma}=\frac{e}{m_{e}^{3}} \sqrt{-\left(F_{\mu \nu} k^{\nu}\right)^{2}},
$$

where $k^{\nu}$ is the four-wave vector on the impinging photon and $F_{\mu \nu}$ is the electromagnetic external field tensor. Natural units are adopted with $c=\hbar=1$, and the fields in the electromagnetic field tensor are normalized on the critical QED field $E_{S}$ (which is given in dimensional units by $E_{S}=$ $m_{e}^{2} c^{3} / e \hbar$ [41]). In the limit of small $\chi_{\gamma}$, for parallel polarizations the square of the photon invariant mass $m$ can be written as

$$
\begin{aligned}
\mathfrak{m}^{2}= & -\alpha m_{e}^{2}\left\{\frac{4}{45 \pi}\left[\chi_{\gamma}^{2}+\frac{1}{3} \chi_{\gamma}^{4}\right]\right. \\
& \left.+i \frac{1}{4} \sqrt{\frac{3}{4}} \chi_{\gamma} \exp \left(-\frac{2}{3 \chi_{\gamma}}\right)+\mathcal{O}\left(\chi_{\gamma}^{6}\right)\right\},
\end{aligned}
$$

where $\alpha=e^{2} / \hbar c$ is the fine structure constant. The imaginary part is exponentially small. The term proportional to $\chi_{\gamma}^{4}$ describes the dispersion effects, i.e., the effects corresponding to the wave propagation velocity dependence on the wave vector. This result was used (to leading order in $\chi_{\gamma}^{4}$ ) in Ref. [36] to derive a nonlinear wave equation for finite amplitude dispersive waves counterpropagating with respect to a cross-field configuration. This wave equation is of the form of the Korteveg-de Vries (KdV) equation [42] in the one-dimensional spatial case and includes third order derivatives of the impinging wave vector potential while it has the form of the KadomtsevPetviashivili equation $[43,44]$ in the two-dimensional case. In the one-dimensional case, in light-cone coordinates $x^{+}, x^{-}$and for normalized variables (for explicit definitions see Sec. III), the KdV equation obtained in Ref. [36] describing the electromagnetic wave in the QED vacuum reads

$$
\partial_{+} a-\left(\kappa_{1}+\kappa_{2} a\right) \partial_{-} a-2 \kappa_{3} \partial_{---} a=0,
$$

where the coefficients $\kappa_{1}=4 \alpha W_{0}^{2} /(45 \pi), \kappa_{2}=32 \sqrt{2} \alpha W_{0}^{3} /$ $(105 \pi)$, and $\kappa_{3}=4 \alpha W_{0}^{4} /(135 \pi)$ are proportional respectively to the second, third, and fourth power of the crossfield amplitude $W_{o}$. The nonlinear term in Eq. (3) arises from the Heisenberg-Euler Lagrangian [3], truncated at the six photon contribution, and allows for propagating Korteveg-de Vries soliton solutions.

In the present article we use a Lagrangian in the electromagnetic vacuum action that involves higher order derivatives of the wave vector potential and that is constructed so as to include the quantum nonlinearity parameter dependency of the invariant photon mass. In this formulation higher order derivatives enter in combination with nonlinear terms. We derive the corresponding field equations by a variation of the action integral and obtain scattering solutions for counterstreaming finite length pulses that include the effect of dispersion. In addition we derive the general solution for finite amplitude waves propagating in a cross-field configuration. These solutions describe a class of soliton solutions of the type described in Ref. [36].

Effective electromagnetic Lagrangians depending on higher derivatives have been introduced in the context of modified linear electrodynamics, or limited to leading order in the field amplitude and field derivatives separately, by Podolsky [45-47], Barut and Mullen [48], Lee and Wick [49], and also see Refs. [50-52]. Essentially these Lagrangians are of the form exemplified by the LeeWick Lagrangian

$$
\begin{aligned}
\mathcal{L} & =\mathcal{L}_{0}+\mathcal{L}_{\mathrm{LW}}, \quad \text { with } \quad \mathcal{L}_{0}=-\frac{1}{4 \pi} F_{\mu \nu} F^{\mu \nu} \text { and } \\
\mathcal{L}_{\mathrm{LW}} & =\frac{1}{4 M^{2}} F_{\mu \nu}\left(\partial^{\alpha} \partial_{\alpha} F^{\mu \nu}\right),
\end{aligned}
$$

with the inclusion, see e.g., Ref. [52], of the first nonlinear contributions from the Heisenberg-Euler Lagrangian. In Eq. (4) $\mathcal{L}_{0}$ is the classical electromagnetic Lagrangian in vacuum and $M$ is a mass parameter. We note that a Lagrangian depending on higher order derivatives of the form

$$
\mathcal{L}_{M M E}=\frac{\alpha}{m_{e}^{2}}\left[-\left(\partial_{\kappa} F_{\lambda}^{\kappa}\right)\left(\partial_{\mu} F^{\mu \nu}\right)+F_{\mu \nu} \partial_{\lambda} \partial^{\lambda} F^{\mu \nu}\right]
$$

was obtained in Refs. [8,9]. A derivative expansion of the effective action for nonlinear quantum electrodynamics has been obtained in Refs. $[53,54]$ in terms of a Lagrangian that is written in the form

$$
\begin{aligned}
\mathcal{L}= & \mathcal{L}_{\mathrm{HE}}+\partial_{\lambda} F_{\alpha \beta} \partial_{\gamma} F_{\sigma \delta} L_{1}^{\lambda \alpha \beta \gamma \sigma \delta}\left(F^{\mu \nu}\right) \\
& + \text { higher field derivative terms }
\end{aligned}
$$

where $\mathcal{L}_{\mathrm{HE}}$ is the Heisenberg-Euler Lagrangian and $L_{1}^{\lambda \alpha \beta \gamma \sigma \delta}\left(F^{\mu \nu}\right)$ is a local function of the electromagnetic field tensor.

\section{A. Well posedness of higher order Lagrangians}

The physical interpretation of higher order derivative Lagrangians presents some difficulties as these Lagrangians lead to "ghost" degrees of freedom and to instabilities. In 1850 Ostrogradski [55] proved in the context of classical mechanics that a Lagrangian of the form $\mathcal{L}(q, \dot{q}, \ddot{q})$, which requires four initial conditions and thus involves four canonical variables, leads to a 
Hamiltonian that is not bounded from below with respect to a "ghost" degree of freedom; see Ref. [56] where it was shown that in a non-degenerate higher derivative theory, that is in a theory where the higher order derivative does not simply amount to a total time derivative, the Ostrogradski instability can only be removed by the addition of constraints that reduce the phase space of the original theory.

For systems with infinite degrees of freedom, higher order derivative Lagrangians can lead to additional wave branches corresponding, e.g., for the Lee-Wick Lagrangian, to two independent (on-shell) spin-1 fields: the original massless photon field and an additional massive one.

In the case of the Podolsky electrodynamics [45-47], as well as in the case considered by Mamaev, Mostepanenko, and Eidis [8], which was introduced in order to regularize the electromagnetic field behavior at short distances and to take into account the field inhomogeneity effects, the Lagrangian can be written as

$$
\begin{aligned}
\mathcal{L} & =\mathcal{L}_{0}+\mathcal{L}_{\text {Pod }}, \quad \text { with } \\
\mathcal{L}_{\text {Pod }} & =\frac{1}{M^{2}}\left[-\left(\partial_{\kappa} F_{\lambda}^{\kappa}\right)\left(\partial_{\mu} F^{\mu \nu}\right)+F_{\mu \nu} \partial_{\lambda} \partial^{\lambda} F^{\mu \nu}\right],
\end{aligned}
$$

where the inverse mass $1 / M$ is the parameter that corresponds to the length, and the current source term is not included. From this Lagrangian we obtain in the Lorentz gauge $\partial_{\mu} A^{\mu}=0$ the wave equation

$$
\left(1-\frac{1}{M^{2}} \partial_{\mu} \partial^{\mu}\right) \partial_{\nu} \partial^{\nu} A^{\kappa}=0 .
$$

It corresponds to a photon branch with dispersion equation $k_{\nu} k^{\nu}=0$ and a ghost branch with dispersion equation $k_{\mu} k^{\mu}=-M^{2}$. The addition of nonlinear terms in the Lagrangian will in general couple the two different branches (see Appendix C). An analogous result can be derived from the Lagrangians introduced in $[48,49]$.

For the sake of consistency, see also Ref. [57], in what follows we will treat the terms with the higher derivatives as corrections to the classical electromagnetic Lagrangian density $\mathcal{L}_{0}$ and in particular we will require that any effective mass arising from the balance between the nonlinear and the dispersive terms remains finite in the limit in which the value of the fine structure constant $\alpha$ is set equal to zero.

\section{B. Outline of the article}

In Sec. II, after specifying for the sake of clarity some normalization conditions, the structure of the dispersive Lagrangian term is formalized for an electromagnetic vacuum configuration in four-dimensional Minkowski space. Its reduced expression in a spatially one-dimensional configuration is then expressed in terms of light-cone variables. In Sec. III the electromagnetic field equations are derived from the full one-dimensional Lagrangian in vacuum including the Heisenberg-Euler and the dispersion terms up to the sixth power of the fields. Then explicit solutions are obtained for the scattering of two counterpropagating finite length pulses in Sec. IV, and for finite amplitude waves in a constant cross-field configuration in Sec. V. These sections are supplemented by four appendixes at the end of the article. The first two appendixes serve the purpose of providing algebraic developments separately so as not to interrupt the flow of the presentation. In the final two appendixes, ghost solutions are described in the case of a nonlinear wave equation with linear dispersion terms derived from the Lagrangian given by Eq. (5), Appendix $\mathrm{C}$, and of the wave equation derived from the Lagrangian given by Eq. (23) for a spacial class of selfsimilar solutions, Appendix D. Finally in Sec. VI conclusions are drawn and a possible inclusion of higher powers of the field amplitudes in the Lagrangian is indicated as a possible path towards the identification of higher order solitonic structures in the process of light-light interaction.

\section{DISPERSIVE CONTRIBUTION TO THE LAGRANGIAN DENSITY}

We specify the normalizations adopted in this article by writing the classical electrodynamics Lagrangian in the form

$$
\mathcal{L}_{0}=-\frac{m_{e}^{4}}{16 \pi \alpha} F_{\mu \nu} F^{\mu \nu}=-\frac{m_{e}^{4}}{4 \pi \alpha} \mathfrak{F}
$$

while the Heisenberg-Euler Lagrangian density (truncated at the 6-photon interaction term) is written as

$\mathcal{L}_{\mathrm{HE}}=-\frac{m_{e}^{4} e^{4}}{90 \pi^{2}}\left[\left(\mathfrak{F}^{2}+\frac{7}{4} \mathfrak{G S}^{2}\right)+\frac{8}{7} \mathfrak{F}\left(\mathfrak{F}^{2}+\frac{13}{16} \mathfrak{G S}^{2}\right)\right]$.

The Lorentz invariants $\mathfrak{F}$ and $\mathfrak{G}$ are defined by

$$
\mathfrak{F}=\frac{1}{4} F_{\mu \nu} F^{\mu \nu}, \quad \mathfrak{S}=-\frac{1}{8} \varepsilon^{\mu \nu \kappa \lambda} F_{\kappa \lambda} F_{\mu \nu},
$$

with $\varepsilon^{\mu \nu \kappa \lambda} F_{\kappa \lambda}$ the dual electromagnetic field tensor. Here $\varepsilon^{\mu \nu \kappa \lambda}$ is the fully antisymetric four-dimensional Levi-Civita tensor.

Referring to the quantum nonlinearity parameter $\chi_{\gamma}$ that is used in the definition of the invariant photon mass and in view of Eq. (6), we define the following dispersive contribution to the vacuum Lagrangian density:

$$
\mathcal{L}_{\text {Disp }}=\frac{\mu m_{e}^{4}}{4 \pi \alpha}\left[\partial _ { \alpha } h ( \mathfrak { F } , ( \mathfrak { S } ) ] F _ { \beta } ^ { \alpha } F ^ { \beta \gamma } \left[\partial_{\gamma} h(\mathfrak{F},(\mathfrak{S})] .\right.\right.
$$

Here $\mu$ is a coefficient that will be identified as 


$$
\mu=\frac{4}{135 \pi} \alpha
$$

by comparing Eq. (3) derived in terms of the invariant photon mass with the corresponding result given by Eq. (33) in Sec. V, and $h$ is a function of the two Lorentz invariants $\mathfrak{F}$, $\mathfrak{G}$. In the following we expand $h$ in a Taylor series and keep only linear terms in $\mathfrak{F}$ and $\mathfrak{G}$.

\section{A. One-dimensional, single polarization case}

In the case of an electromagnetic configuration that depends on a single spatial coordinate, say $x$, and where the fields correspond to a single polarization state, $\mathbb{G}$ vanishes and Eq. (12) becomes

$$
\begin{aligned}
\mathcal{L}_{\text {Disp } \|}= & -\frac{\mu m_{e}^{4}}{4 \pi \alpha}\left\{B^{2}\left[\partial_{x}\left(E^{2}-B^{2}\right)\right]^{2}+E^{2}\left[\partial_{t}\left(E^{2}-B^{2}\right)\right]^{2}\right. \\
& \left.-2 E B\left[\partial_{x}\left(E^{2}-B^{2}\right) \partial_{t}\left(E^{2}-B^{2}\right)\right]\right\},
\end{aligned}
$$

while

$$
\begin{aligned}
\mathcal{L}_{0}+\mathcal{L}_{\mathrm{HE}}= & -\frac{m_{e}^{4}}{4 \pi \alpha}\left[\frac{B^{2}-E^{2}}{2}-\epsilon_{2} \frac{\left(B^{2}-E^{2}\right)^{2}}{4}\right. \\
& \left.+\epsilon_{3} \frac{\left(B^{2}-E^{2}\right)^{3}}{8}\right],
\end{aligned}
$$

where

$$
\epsilon_{2}=\frac{2 \alpha}{45 \pi} \quad \text { and } \quad \epsilon_{3}=\frac{32 \alpha}{315 \pi} .
$$

\section{ACTION FUNCTIONAL IN LIGHT-CONE VARIABLES}

Here as in previous articles, see e.g., Refs. [36,58-60], for a spatially one-dimensional configuration it is convenient to introduce light-cone variables defined as

$$
x^{+}=(x+t) / \sqrt{2}, \quad x^{-}=(x-t) / \sqrt{2},
$$

with corresponding derivatives

$$
\partial_{x}=\left(\partial_{+}+\partial_{-}\right) / \sqrt{2}, \quad \partial_{t}=\left(\partial_{+}-\partial_{-}\right) / \sqrt{2},
$$

where $\partial_{ \pm}=\partial / \partial x^{ \pm}$. For a configuration corresponding to a single transverse polarization state we choose the fourvector potential to have only a component in the $z$ direction which we denote in normalized form by $a\left(x^{+}, x^{-}\right)$. Then the electric field $E$ is in the $z$ direction while the magnetic field $B$ is along $y$.

We define the field variables

$$
w\left(x^{+}, x^{-}\right)=\partial_{+} a\left(x^{+}, x^{-}\right), \quad u\left(x^{+}, x^{-}\right)=\partial_{-} a\left(x^{+}, x^{-}\right),
$$

i.e., the electric and magnetic fields are

$$
E=(u-w) / \sqrt{2}, \quad B=-(u+w) / \sqrt{2} .
$$

Note that by construction we recover Faraday's law in the form

$$
\partial_{-} w=\partial_{-+} a=\partial_{+} u \text {. }
$$

By including both $\mathcal{L}_{0}+\mathcal{L}_{\mathrm{HE}}$ and $\mathcal{L}_{\mathrm{Disp} \|}$, the electromagnetic action $\mathcal{A}$ expressed in the $x^{+}, x^{-}, w, u$ variables takes the form

$$
\mathcal{A}(a)=\frac{m^{4}}{4 \pi \alpha} \iint_{\mathcal{D}} d x^{+} d x^{-} \mathcal{L}_{T}\left(a, a^{\prime}, a^{\prime \prime}\right),
$$

where $a^{\prime}$ stands symbolically for $w=\partial_{+} a$ and $u=\partial_{-} a$, while $a^{\prime \prime}$ stands for $\partial_{+} w=\partial_{++} a, \partial_{-} w=\partial_{+} u=\partial_{+-} a$, and $\partial_{-} u=\partial_{--} a$. After eliminating the common multiplicative factor in Eqs. (14) and (15) the Lagrangian $\mathcal{L}_{T}$ reads

$$
\begin{aligned}
\mathcal{L}_{T}\left(a, a^{\prime}, a^{\prime \prime}\right)= & -u w+\epsilon_{2}(u w)^{2}-\epsilon_{3}(u w)^{3} \\
& -\mu\left\{w^{2}\left[\partial_{-}(u w)\right]^{2}+u^{2}\left[\partial_{+}(u w)\right]^{2}\right. \\
& \left.+2 u w\left[\partial_{+}(u w)\right]\left[\partial_{-}(u w)\right]\right\} .
\end{aligned}
$$

The dependence of Lagrangian $\mathcal{L}_{T}$ on the first and second order derivatives of the vector potential $a\left(x^{+}, x^{-}\right)$ can be made explicit by rewriting (see Appendix A) $\mathcal{L}_{T}\left(a, a^{\prime}, a^{\prime \prime}\right)$ as

$$
\begin{aligned}
\mathcal{L}_{T}\left(a, a^{\prime}, a^{\prime \prime}\right)= & -\left(\partial_{+} a\right)\left(\partial_{-} a\right)+\epsilon_{2}\left[\left(\partial_{+} a\right)\left(\partial_{-} a\right)\right]^{2} \\
& -\epsilon_{3}\left[\left(\partial_{+} a\right)\left(\partial_{-} a\right)\right]^{3}-\mu\left[\left(\partial_{+} a\right)^{2}\left(\partial_{--} a\right)\right. \\
& \left.+2\left(\partial_{-} a\right)\left(\partial_{+} a\right)\left(\partial_{+-} a\right)+\left(\partial_{-} a\right)^{2}\left(\partial_{++} a\right)\right]^{2} .
\end{aligned}
$$

Varying the action $\mathcal{A}(a)$ with respect to the vector potential $a$ and imposing that at the boundaries $\delta \mathcal{D}$ of the domain $\mathcal{D}$ under consideration both $\delta a$ and $\partial_{+} \delta a, \partial_{-} \delta a$ vanish, we obtain the wave equation for the vector potential

$$
\begin{gathered}
-\partial_{+} \frac{\partial \mathcal{L}_{T}}{\partial\left(\partial_{+} a\right)}-\partial_{-} \frac{\partial \mathcal{L}_{T}}{\partial\left(\partial_{-} a\right)}+\partial_{++} \frac{\partial \mathcal{L}_{T}}{\partial\left(\partial_{++} a\right)} \\
+\partial_{--} \frac{\partial \mathcal{L}_{T}}{\partial\left(\partial_{--} a\right)}+\partial_{+-} \frac{\partial \mathcal{L}_{T}}{\partial\left(\partial_{+-} a\right)}=0
\end{gathered}
$$

where $\quad \partial_{+} a=w, \partial_{-} a=u \quad$ and $\quad \partial_{++} a=\partial_{+} w, \partial_{+-}=$ $\partial_{+} u=\partial_{-} w, \partial_{--} a=\partial_{-} u$ are treated as independent variables in the differentiation. The explicit form of the derivatives of $\mathcal{L}_{T}$ in Eq. (25) are given in Appendix B. 


\section{A. Field equations in the $u, w$ variables}

The Lagrangian $\mathcal{L}_{T}(w, u)$ in Eq. (23) can be rewritten as

$$
\mathcal{L}_{T}(u, w)=-\left[u w-\epsilon_{2}(u w)^{2}+\epsilon_{3}(u w)^{3}+\mu\left(w^{2} \partial_{-} u+u w \partial_{+} u+u w \partial_{-} w+u^{2} \partial_{+} w\right)^{2}\right],
$$

and the wave equation for the vector potential (25) can be written in terms of the field variables $u$ and $w$ as

$$
\begin{aligned}
\partial_{+} & \left\{u\left[1-2 \epsilon_{2} u w+3 \epsilon_{3}(u w)^{2}\right]\right\}+\partial_{-}\left\{w\left[1-2 \epsilon_{2} u w+3 \epsilon_{3}(u w)^{2}\right]\right\} \\
& +4 \mu\left\{\partial_{+}\left[w\left(\partial_{-}(u w)\right)^{2}+u \partial_{+}(u w) \partial_{-}(u w)\right]+\partial_{-}\left[u\left(\partial_{+}(u w)\right)^{2}+w \partial_{+}(u w) \partial_{-}(u w)\right]\right\} \\
& -2 \mu\left\{\partial_{++}\left[u^{3} \partial_{+}(u w)+u^{2} w \partial_{-}(u w)\right]+\partial_{--}\left[w^{3} \partial_{-}(u w)+u w^{2} \partial_{+}(u w)\right]+2 \partial_{+-}\left[u w^{2} \partial_{-}(u w)+u^{2} w \partial_{+}(u w)\right]\right\},
\end{aligned}
$$

which can be derived by rearranging Eq. (B3) in Appendix B.

\section{SCATTERING SOLUTIONS FOR COUNTERSTREAMING FINITE LENGTH PULSES}

The asymptotic effect of the interaction between two counterpropagating electromagnetic pulses with a finite length can be derived directly from Eq. (25) assuming that for large $\left|x_{ \pm}\right|$there is no superposition between the pulses so that the vector potential $a\left(x^{+}, x^{-}\right)$can be written as $a\left(x^{+}, x^{-}\right)=a_{+}\left(x^{+}\right)+a_{-}\left(x^{-}\right)$. Integrating Eq. (25) over $x^{+}$we obtain

$$
\left.\frac{\partial \mathcal{L}_{T}}{\partial\left(\partial_{+} a\right)}\right|_{-\infty} ^{+\infty}=-\partial_{-} \int_{-\infty}^{+\infty} d x^{+} \frac{\partial \mathcal{L}_{T}}{\partial\left(\partial_{-} a\right)}+\partial_{--} \int_{-\infty}^{+\infty} d x^{+} \frac{\partial \mathcal{L}_{T}}{\partial\left(\partial_{--} a\right)}
$$

Then, considering a perturbative expansion around $a_{0}\left(x^{+}, x^{-}\right)=a_{0+}\left(x^{+}\right)+a_{0-}\left(x^{-}\right)$, and using Eq. (B1), we find

$$
\begin{aligned}
\left.\partial_{-} a\right|_{-\infty} ^{+\infty}= & \partial_{-} \int_{-\infty}^{+\infty} d x^{+}\left[\epsilon_{2}\left(\partial_{+} a_{0}\right)^{2}\left(\partial_{-} a_{0}\right)-\frac{3}{2} \epsilon_{3}\left(\partial_{+} a_{0}\right)^{3}\left(\partial_{-} a_{0}\right)^{2}-\mu\left(\partial_{-} a_{0}\right)^{3}\left(\partial_{++} a_{0}\right)^{2}\right] \\
& +2 \mu \partial_{--} \int_{-\infty}^{+\infty} d x^{+}\left(\partial_{+} a_{0}\right)^{4}\left(\partial_{--} a_{0}\right) .
\end{aligned}
$$

The terms on the right-hand side arise from the Heisenberg-Euler Lagrangian (the $\epsilon_{2}, \epsilon_{3}$ terms) and from the dispersive additional contribution (the $\mu$ terms) given in Eq. (14). In deriving Eq. (29) we used the fact that $\partial_{+-} a_{0}=0$ and that two terms proportional to $\left(\partial_{+} a_{0}\right)^{2}\left(\partial_{++} a_{0}\right)$ are total derivatives with respect to $x^{+}$and thus do not contribute to the $x^{+}$integral when asymptotically there is no superposition between the pulses. The term on the left-hand side represents the lowest order change of the vector potential pulse propagating along the positive $x$ direction due to the scattering with the counterpropagating pulse, and the factor 2 in front of it arises from the contribution of the classical electromagnetic Lagrangian. Expressed in terms of the electromagnetic field variables Eq. (29) reads

$$
\begin{aligned}
\left.u\right|_{-\infty} ^{+\infty}= & \epsilon_{2}\left(\partial_{-} u_{0}\right) \int_{-\infty}^{+\infty} d x^{+} w_{0}^{2}-\frac{3}{2} \epsilon_{3}\left(\partial_{-} u_{0}^{2}\right) \int_{-\infty}^{+\infty} d x^{+} w_{0}^{3}-\mu\left(\partial_{-} u_{0}^{3}\right) \int_{-\infty}^{+\infty} d x^{+}\left(\partial_{+} w_{0}\right)^{2} \\
& +\mu\left(\partial_{---} u_{0}\right) \int_{-\infty}^{+\infty} d x^{+} w_{0}^{4} .
\end{aligned}
$$

where $w_{0}=w_{0}\left(x^{+}\right)=\partial_{+} a_{0}\left(x^{+}\right) \quad$ and $\quad u_{0}=u_{0}\left(x^{-}\right)=$ $\partial_{-} a_{0}\left(x^{-}\right)$. A corresponding equation can be derived for $\left.w\right|_{-\infty} ^{+\infty}$. The first term on the right-hand side of Eq. (30) corresponds to the standard phase shift due to reduced propagation velocity during the interaction phase $[59,61,62]$ while the second, if the integral of $w_{0}^{3}$ does not vanish, to the six-photon interaction contribution to the harmonic generation mechanism discussed e.g., in Ref. [63]. The third terms corresponds to a new harmonic generation process that depends on the square of the derivative of the field amplitude of the counterpropagating pulse, while the fourth term provides a dispersion correction to the phase shift given by the first term and corresponds to a widening of the pulse.

\section{SOLUTIONS IN CONSTANT CROSS FIELDS}

The system of Eqs. (21) and (27) admits solutions in the form of the progressive nonlinear waves that propagate with constant "velocity" $S>0$, i.e., with functions $u$ and $w$ that depend on the variable

$$
\psi=x^{-}+S x^{+}=\frac{1}{\sqrt{2}}[x(1+S)-t(1-S)] .
$$


In the $x-t$ variables the wave propagates with the velocity equal to $(1-S) /(1+S)$.

Using Eq. (21) we obtain a relationship between $u(\psi)$ and $w(\psi)$

$$
w=S u+W_{0},
$$

where $W_{0}$ is constant which corresponds to a constant cross-field configuration with equal amplitude electric and magnetic fields, $E_{0}=B_{0}=\sqrt{2} W_{0}$. The Poynting vector $c \mathbf{E} \times \mathbf{B} / 4 \pi$ of the cross-field configuration that is taken to model a low frequency wave is directed in the negative direction along the $x$ axis so that $\mathbf{E}=\mathbf{e}_{z} E_{0}, \mathbf{B}=\mathbf{e}_{y} B_{0}$, where $\mathbf{e}_{y}$ and $\mathbf{e}_{z}$ are unit vectors in the $y$ and $z$ directions. The high frequency electromagnetic wave described by the variables $w$ and $u$ in Eq. (32) propagates in the positive direction along the $x$ axis.

Assuming for the sake of simplicity that the amplitude of the high frequency wave amplitude is much smaller than the cross-field amplitude $\left(|u|,|w| \ll W_{0}\right)$ we obtain from Eqs. (27) and (32)

$$
\begin{aligned}
2 \mu W_{0}^{4} \partial_{---} u= & \partial_{+}\left(u-2 \epsilon_{2} W_{0} u^{2}\right) \\
& +\partial_{-}\left(S u-2 \epsilon_{2} W_{0}^{2} u+3 \epsilon_{3} W_{0}^{3} u^{2}\right) .
\end{aligned}
$$

Using the ansatz (31) we obtain

$$
\mu W_{0}^{4} u^{\prime \prime \prime}=\left[S-\epsilon_{2} W_{0}^{2}-\left(2 S \epsilon_{2} W_{0}-3 \epsilon_{3} W_{0}^{3}\right) u\right] u^{\prime},
$$

where a prime stands for differentiation with respect to $\psi$. This is the well-known Korteveg-de Vries equation for the stationary nonlinear wave propagating with constant velocity $S$ (see Refs. [42,64,65] and Ref. [36] for the case of the $\mathrm{KdV}$ solitons in the QED vacuum). Integration of Eq. (34) over $\psi$ yields

$\mu W_{0}^{4} u^{\prime \prime}=\left(S-\epsilon_{2} W_{0}^{2}\right) u-\left(S \epsilon_{2} W_{0}-3 \epsilon_{3} W_{0}^{3} / 2\right) u^{2}+C_{1}$.

Multiplying this equation on $u^{\prime}$ and integrating over $\psi$ yields

$$
\begin{aligned}
\mu W_{0}^{4}\left(u^{\prime}\right)^{2}= & \left(S-\epsilon_{2} W_{0}^{2}\right) u^{2}-\left(2 S \epsilon_{2} W_{0} / 3-\epsilon_{3} W_{0}^{3}\right) u^{3} \\
& +2 C_{1} u+C_{2},
\end{aligned}
$$

where $C_{1}$ and $C_{2}$ are constants.

Choosing $C_{1}=C_{2}=0$ we find the solution of Eq. (36) in the form of a $\mathrm{KdV}$ soliton. It reads

$$
\begin{aligned}
u\left(x^{+}+S x^{-}\right)= & \frac{3\left(S-\epsilon_{2} W_{0}^{2}\right)}{\left(2 \epsilon_{2} W_{0}-3 \epsilon_{3} W_{0}^{3}\right)} \cosh ^{-2} \\
& \times\left[\frac{\sqrt{S-\epsilon_{2} W_{0}^{2}}\left(x^{+}+S x^{-}\right)}{2 \sqrt{\mu W_{0}^{4}}}\right] .
\end{aligned}
$$

The soliton amplitude $u_{0}$ and width $l_{0}$ are given by

$u_{0}=\frac{3\left(S-\epsilon_{2} W_{0}^{2}\right)}{\left(2 \epsilon_{2} W_{0}-3 \epsilon_{3} W_{0}^{3}\right)} \quad$ and $\quad l_{0}=2 \sqrt{\frac{\mu W_{0}^{4}}{S-\epsilon_{2} W_{0}^{2}}}$.

We see that the parameter $S$ determining the soliton propagation velocity depends on the soliton amplitude $u_{0}$ as

$$
S=\epsilon_{2} W_{0}^{2}+\left(\frac{2}{3} \epsilon_{2} W_{0}-\epsilon_{3} W_{0}^{3}\right) u_{0} .
$$

The soliton propagation velocity depends on $W_{0}$ and $u_{0}$ as

$$
\begin{aligned}
V & =\frac{1-S}{1+S}=\frac{1-\epsilon_{2} W_{0}^{2}-\left(2 \epsilon_{2} / 3-\epsilon_{3} W_{0}^{2}\right) W_{0} u_{0}}{1+\epsilon_{2} W_{0}^{2}+\left(2 \epsilon_{2} / 3-\epsilon_{3} W_{0}^{2}\right) W_{0} u_{0}} \\
& \approx 1-2 \epsilon_{2} W_{0}^{2}-\frac{4}{3} \epsilon_{2} W_{0} u_{0} .
\end{aligned}
$$

Substituting $S$ from Eq. (39) to the expression for $l_{0}$ given by Eq. (38) we find the soliton width. It reads

$$
l_{0}=\sqrt{\frac{4 \mu W_{0}^{3}}{\left(2 \epsilon_{2}-3 \epsilon_{3} W_{0}^{2}\right) u_{0}}},
$$

$W_{0}<\left(2 \epsilon_{2} / 3 \epsilon_{3}\right)$, i.e., $l_{0} \approx \chi_{C}\left(W_{0}^{3} / u_{0}\right)^{1 / 2}$. In other words a typical energy of the photons constituting the soliton is approximately equal to $\hbar \omega_{\gamma} \approx m_{e} c^{2}\left(W_{0}^{3} / u_{0}\right)^{1 / 2}$.

\section{CONCLUSIONS}

We use a Lagrangian that involves higher order derivatives of the wave vector potential and that is constructed so as to include the quantum nonlinearity parameter dependency of the invariant photon mass. This Lagrangian allows us to describe dispersive effects in the interaction of two counterpropagating light pulses by a nonlocal extension of the nonlinear wave equation that is derived from the Heisenberg-Euler Lagrangian. In addition, in the case of a finite amplitude wave impinging on large cross fields, we show that Korteveg-de Vries soliton solutions can be consistently derived from these field equations by considering a proper ordering of the amplitude of the impinging wave and of its space-time coordinate dependence in terms of the amplitude of the cross fields.

An extension of this procedure so as to include higher order derivatives and higher powers of the fields amplitude than those considered in this article could be of interest when searching for novel light soliton solutions, such as e.g., "compactons," i.e., solitons with finite wavelength [66]. Such an extension could be written in the formal way 


$$
\begin{aligned}
\mathcal{L}_{H E / D}= & \frac{m^{4}}{4 \pi \alpha} \epsilon_{2} h\left(F_{\mu \nu} F^{\mu \nu}, \epsilon^{\mu \nu \kappa \lambda} F_{\mu \nu} F_{\kappa \lambda}\right) \\
& \times\left[1+f\left(\overleftarrow{\partial_{\alpha}} F_{\beta}^{\alpha} F^{\beta \gamma} \overrightarrow{\partial_{\gamma}}\right)\right] h\left(F_{\mu \nu} F^{\mu \nu}, \epsilon^{\mu \nu \kappa \lambda} F_{\mu \nu} F_{\kappa \lambda}\right),
\end{aligned}
$$

where the function $h$ is related to the usual HeisenbergEuler asymptotic expansion while $f$ is a "function" of the differential operator $\overleftarrow{\partial_{\alpha}} F_{\beta}^{\alpha} F^{\beta \gamma} \overrightarrow{\partial_{\gamma}}$ (related to the relativistic $\chi$ invariant) where the arrows indicate left or right action. The function $f$ should be related to the expansion of the invariant photon mass in Eq. (2), see Ref. [31]. The Lagrangian $\mathcal{L}_{H E / D}$ is gauge invariant and is Lorentz invariant. In addition, its contribution to the wave equation vanishes in the case of a plane wave in which case $F_{\mu \nu} F^{\mu \nu}=\varepsilon^{\mu \nu \kappa \lambda} F_{\mu \nu} F_{\kappa \lambda}=0$.

\section{ACKNOWLEDGMENTS}

The work is supported by the project High Field Initiative (CZ.02.1.01/0.0/0.0/15_003/0000449) from the European Regional Development Fund.

\section{APPENDIX A: EXPLICIT FORM OF THE LAGRANGIAN $\mathcal{L}_{T S}$}

We rewrite the Lagrangian $\mathcal{L}_{T}$ in Eq. (23) explicitly as a function of $\partial_{+} a, \partial_{-} a, \partial_{++} a, \partial_{+-} a, \partial_{--} a$,

$$
\begin{aligned}
\mathcal{L}_{T}\left(a, a^{\prime}, a^{\prime \prime}\right)= & -\left\{\left(\partial_{+} a\right)\left(\partial_{-} a\right)-\epsilon_{2}\left[\left(\partial_{+} a\right)\left(\partial_{-} a\right)\right]^{2}+\epsilon_{3}\left[\left(\partial_{+} a\right)\left(\partial_{-} a\right)\right]^{3}\right. \\
& +\mu\left[\left(\partial_{+} a\right)^{2}\left(\left(\partial_{--} a\right)\left(\partial_{+} a\right)+\left(\partial_{-} a\right)\left(\partial_{+-} a\right)\right)^{2}+\left(\partial_{-} a\right)^{2}\left(\left(\partial_{-+} a\right)\left(\partial_{+} a\right)+\left(\partial_{-} a\right)\left(\partial_{++} a\right)\right)^{2}\right. \\
& \left.\left.+2\left(\partial_{-} a\right)\left(\partial_{+} a\right)\left(\left(\partial_{--} a\right)\left(\partial_{+} a\right)+\left(\partial_{-} a\right)\left(\partial_{+-} a\right)\right)\left(\left(\partial_{-+} a\right)\left(\partial_{+} a\right)+\left(\partial_{-} a\right)\left(\partial_{++} a\right)\right)\right]\right\},
\end{aligned}
$$

which can be rewritten as

$$
\begin{aligned}
\mathcal{L}_{T}\left(a, a^{\prime}, a^{\prime \prime}\right) & =-\left\{\left(\partial_{+} a\right)\left(\partial_{-} a\right)-\epsilon_{2}\left(\left(\partial_{+} a\right)\left(\partial_{-} a\right)\right)^{2}+\epsilon_{3}\left(\left(\partial_{+} a\right)\left(\partial_{-} a\right)\right)^{3}\right. \\
& \left.+\mu\left[\left(\left(\partial_{+} a\right)^{2}\left(\partial_{--} a\right)+2\left(\partial_{-} a\right)\left(\partial_{+} a\right)\left(\partial_{+-} a\right)+\left(\partial_{-} a\right)^{2}\left(\partial_{++} a\right)\right)^{2}\right]\right\},
\end{aligned}
$$

and can be reexpressed in terms of the electromagnetic fields as

$$
\mathcal{L}_{T}(w, u)=-\left\{u w-\epsilon_{2}(u w)^{2}+\epsilon_{3}(u w)^{3}+\mu\left[w^{2} \partial_{-} u+u w \partial_{+} u+u w \partial_{-} w+u^{2} \partial_{+} w\right]^{2}\right\} .
$$

\section{APPENDIX B: DERIVATION OF THE FIELD EQUATION}

From Eq. (24) we find

$$
\begin{aligned}
\frac{\partial \mathcal{L}_{T}}{\partial\left(\partial_{+} a\right)}= & -\left(\partial_{-} a\right)\left[1-2 \epsilon_{2}\left(\partial_{+} a\right)\left(\partial_{-} a\right)+3 \epsilon_{3}\left(\partial_{+} a\right)^{2}\left(\partial_{-} a\right)^{2}\right] \\
& -4 \mu\left\{\left[\left(\partial_{+} a\right)\left(\partial_{--} a\right)+\left(\partial_{-} a\right)\left(\partial_{+-} a\right)\right]\left[\left(\partial_{+} a\right)^{2}\left(\partial_{--} a\right)+2\left(\partial_{-} a\right)\left(\partial_{+} a\right)\left(\partial_{+-} a\right)+\left(\partial_{-} a\right)^{2}\left(\partial_{++} a\right)\right]\right\}, \\
\frac{\partial \mathcal{L}_{T}}{\partial\left(\partial_{-} a\right)}= & -\left[\partial_{+} a\right)\left(1-2 \epsilon_{2}\left(\partial_{+} a\right)\left(\partial_{-} a\right)+3 \epsilon_{3}\left(\partial_{+} a\right)^{2}\left(\partial_{-} a\right)^{2}\right] \\
& -4 \mu\left\{\left[\left(\partial_{+} a\right)\left(\partial_{+-} a\right)+\left(\partial_{-} a\right)\left(\partial_{++} a\right)\right]\left[\left(\partial_{+} a\right)^{2}\left(\partial_{--} a\right)+2\left(\partial_{-} a\right)\left(\partial_{+} a\right)\left(\partial_{+-} a\right)+\left(\partial_{-} a\right)^{2}\left(\partial_{++} a\right)\right]\right\}, \\
\frac{\partial \mathcal{L}_{T}}{\partial\left(\partial_{++} a\right)}= & -2 \mu\left\{\left(\partial_{-} a\right)^{2}\left[\left(\partial_{+} a\right)^{2}\left(\partial_{--} a\right)+2\left(\partial_{-} a\right)\left(\partial_{+} a\right)\left(\partial_{+-} a\right)+\left(\partial_{-} a\right)^{2}\left(\partial_{++} a\right)\right]\right\}, \\
\frac{\partial \mathcal{L}_{T}}{\partial\left(\partial_{--} a\right)}= & -2 \mu\left\{\left(\partial_{+} a\right)^{2}\left[\left(\partial_{+} a\right)^{2}\left(\partial_{--} a\right)+2\left(\partial_{-} a\right)\left(\partial_{+} a\right)\left(\partial_{+-} a\right)+\left(\partial_{-} a\right)^{2}\left(\partial_{++} a\right)\right]\right\}, \\
\frac{\partial \mathcal{L}_{T}}{\partial\left(\partial_{+-} a\right)}= & -4 \mu\left\{\left(\partial_{+} a\right)\left(\partial_{-} a\right)\left[\left(\partial_{+} a\right)^{2}\left(\partial_{--} a\right)+2\left(\partial_{-} a\right)\left(\partial_{+} a\right)\left(\partial_{+-} a\right)+\left(\partial_{-} a\right)^{2}\left(\partial_{++} a\right)\right]\right\} .
\end{aligned}
$$

Using Eq. (B1), reintroducing the field variables $u, w$, and defining for the sake of notational compactness

$$
\mathcal{M}(u, w)=w^{2} \partial_{-} u+u w \partial_{-} w+u w \partial_{+} u+u^{2} \partial_{+} w=w \partial_{-}(u w)+u \partial_{+}(u w)
$$


from Eq. (25) we find

$$
\begin{aligned}
& \partial_{+}\left\{u\left[1-2 \epsilon_{2} w u+3 \epsilon_{3}(w u)^{2}\right]\right\}+\partial_{-}\left\{w\left[1-2 \epsilon_{2} w u-3 \epsilon_{3}(w u)^{2}\right]\right\} \\
& +2 \mu\left\{2 \partial_{+}\left[\left(w \partial_{-} u+u \partial_{-} w\right) \mathcal{M}(u, w)\right]+2 \partial_{-}\left[\left(w \partial_{+} u+u \partial_{+} w\right) \mathcal{M}(u, w)\right]\right. \\
& \left.-\partial_{+} \partial_{+}\left[u^{2} \mathcal{M}(u, w)\right]-\partial_{-} \partial_{-}\left[w^{2} M(u, w)\right]-2 \partial_{+} \partial_{-}[u w \mathcal{M}(u, w)]\right\} .
\end{aligned}
$$

\section{APPENDIX C: GHOSTS BRANCHES}

Here we discuss ghost branches of the higher order Lagrangians considered in this article. Implementing the high derivative term (5) of the Lagrangian (10) in the Heisenberg-Euler Lagrangian leads to

$$
\mathcal{L}(u, w)=-u w+\epsilon_{2}(u w)^{2}-\epsilon_{3}(u w)^{3}-\tilde{\mu}\left[\left(\partial_{+} u\right)\left(\partial_{-} w\right)+u \partial_{+-} w+w \partial_{+-} u\right]
$$

where $\tilde{\mu}=M^{-2}$ is proportional to the fine structure constant $\alpha$., leading to the system of wave equations

$$
\begin{gathered}
\partial_{-} w=\partial_{+} u \\
\partial_{+}\left\{u\left[1-2 \epsilon_{2} u w+3 \epsilon_{3}(u w)^{2}\right]\right\}+\partial_{-}\left\{w\left[1-2 \epsilon_{2} u w+3 \epsilon_{3}(u w)^{2}\right]\right\}+4 \tilde{\mu} \partial_{++-} u .
\end{gathered}
$$

We consider an electromagnetic wave counterpropagating with respect to a large amplitude cross-field low frequency electromagnetic wave, i.e., we assume that

$$
a\left(x^{+}, x^{-}\right)=W_{0} x^{+}+\tilde{a}\left(x^{+}, x^{-}\right) .
$$

Within the linear wave approximation, which requires $\left\{\left|\partial_{-} \tilde{a}\right|,\left|\partial_{+} \tilde{a}\right|\right\} \ll W_{0}$, Eqs. (C2) and (C4) can be reduced to the equation

$$
\partial_{+} \tilde{a}-\epsilon_{2} W_{0}^{2} \partial_{-} \tilde{a}+4 \tilde{\mu} \partial_{++-} \tilde{a}=0
$$

which can be rewritten in $x, t$ variables as

$$
\begin{aligned}
(1 & \left.+\epsilon_{2} W_{0}^{2}\right) \partial_{t} \tilde{a}+\left(1-\epsilon_{2} W_{0}^{2}\right) \partial_{x} \tilde{a}+2 \tilde{\mu}\left(\partial_{t}+\partial_{x}\right) \\
& \times\left(\partial_{t t}-\partial_{x x}\right) \tilde{a}=0
\end{aligned}
$$

The corresponding dispersion equation giving a relationship between the wave frequency $\omega$ and wave number $k$, i.e., $\tilde{a}(x, t) \propto \exp (-i(\omega t-k x))$, has a form

$$
\left(1+\epsilon_{2} W_{0}^{2}\right) \omega-\left(1-\epsilon_{2} W_{0}^{2}\right) k-2 \tilde{\mu}(\omega-k)\left(\omega^{2}-k^{2}\right)=0 .
$$

It is convenient to rewrite this equation in terms of $\Omega=$ $(\omega+k) / \sqrt{2}$ and $Q=(\omega-k) / \sqrt{2}$. In this case, we have $\tilde{a}\left(x^{+}, x^{-}\right) \propto \exp \left(-i\left(\Omega x^{+}+Q x^{-}\right)\right)$. The dispersion equation can be written as

$$
\Omega-\epsilon_{2} W_{0}^{2} Q+4 \tilde{\mu} \Omega^{2} Q,=0,
$$

whose solution gives for two branches

$$
\Omega_{ \pm}=\frac{-1 \pm \sqrt{1+16 \epsilon_{2} W_{0}^{2}}}{8 \tilde{\mu} Q}
$$

In the long-wavelength limit $Q \rightarrow 0$ and/or in the limit of week dispersion $\tilde{\mu} \rightarrow 0$ the frequency $\Omega_{+}$equals

$$
\Omega_{+}=\epsilon_{2} W_{0}^{2} Q-4 \tilde{\mu} \epsilon_{2} W_{0}^{4} Q^{3}+\cdots,
$$

while the frequency $\Omega_{-}$corresponds to the ghost branch,

$$
\Omega_{-}=\frac{1}{4 \tilde{\mu} Q}+\epsilon_{2} W_{0}^{2} Q \ldots
$$

In terms of $\omega_{-}$and $k_{-}$this results in the dispersion equation

$$
\omega_{-}=\sqrt{k_{-}^{2}+\frac{1}{4 \tilde{\mu}}},
$$

i.e., it describes the photons with the "mass" which tends to infinity when $\tilde{\mu} \rightarrow 0$. Using Eq. (13) we find that the electromagnetic field with the photon energy corresponding to the mass, $\hbar \omega_{-}=\sqrt{135 \pi / 4 \alpha} m_{e} c^{2}$ which cannot be described within the Heisenberg-Euler Lagrangian paradigm in contrast to the the wave corresponding to the branch given by Eq. (C10).

\section{APPENDIX D: LORENTZ INVARIANT SOLUTIONS}

Following a procedure adopted in Ref. [59] we may look for self-similar solutions where the vector potential $a\left(x^{+}, x^{-}\right)$depends only on the combination $x^{+} x^{-}=\rho$ which is invariant under Lorentz boosts along $x$. 
Using the following formulas for Lorentz invariant solutions with $a\left(x^{+}, x^{-}\right)=\hat{a}(\rho)$ and $\hat{a}^{\prime}=d \hat{a} / d \rho$

$$
\begin{gathered}
\partial_{+} a=x^{-} \hat{a}^{\prime}, \quad \partial_{-} a=x^{+} \hat{a}^{\prime}, \rho\left(\hat{a}^{\prime}\right)^{2}=u w, \quad \partial_{++} a=\left(x^{-}\right)^{2} \hat{a}^{\prime \prime}, \quad \partial_{--} a=\left(x^{+}\right)^{2} \hat{a}^{\prime \prime}, \quad \partial_{+-} a=\hat{a}^{\prime}+\rho \hat{a}^{\prime \prime} \\
\left(\partial_{+} a\right)^{2}\left(\partial_{--} a\right)+2\left(\partial_{-} a\right)\left(\partial_{+} a\right)\left(\partial_{+-} a\right)+\left(\partial_{-} a\right)^{2}\left(\partial_{++} a\right)=4 \rho^{2}\left(\hat{a}^{\prime}\right)^{2} \hat{a}^{\prime \prime}+2 \rho\left(\hat{a}^{\prime}\right)^{3},
\end{gathered}
$$

we obtain

$$
\begin{aligned}
\frac{\partial \mathcal{L}_{T}}{\partial\left(\partial_{+} a\right)} & =-x^{+}\left[\hat{a}^{\prime}-2 \epsilon_{2} \rho\left(\hat{a}^{\prime}\right)^{3}+3 \epsilon_{3} \rho^{2}\left(\hat{a}^{\prime}\right)^{5}+4 \mu\left(\left(\hat{a}^{\prime}\right)^{2}+2 \rho \hat{a}^{\prime} \hat{a}^{\prime \prime}\right)\left(4 \rho^{2}\left(\hat{a}^{\prime}\right)^{2} \hat{a}^{\prime \prime}+2 \rho\left(\hat{a}^{\prime}\right)^{3}\right)\right] \\
& =-x^{+}\left[\hat{a}^{\prime}-2 \epsilon_{2} \rho\left(\hat{a}^{\prime}\right)^{3}+3 \epsilon_{3} \rho^{2}\left(\hat{a}^{\prime}\right)^{5}\right]-8 \mu x^{+} \rho \hat{a}^{\prime}\left[\left(\rho\left(a^{\prime}\right)^{2}\right)^{\prime}\right]^{2} \\
\frac{\partial \mathcal{L}_{T}}{\partial\left(\partial_{-} a\right)} & =-x^{-}\left[\hat{a}^{\prime}-2 \epsilon_{2} \rho\left(\hat{a}^{\prime}\right)^{3}+3 \epsilon_{3} \rho^{2}\left(\hat{a}^{\prime}\right)^{5}\right]-8 \mu x^{-} \rho \hat{a}^{\prime}\left[\left(\rho\left(a^{\prime}\right)^{2}\right)^{\prime}\right]^{2} \\
\frac{\partial \mathcal{L}_{T}}{\partial\left(\partial_{++} a\right)} & =-2 \mu\left(x^{+}\right)^{2}\left(\hat{a}^{\prime}\right)^{2}\left(4 \rho^{2}\left(\hat{a}^{\prime}\right)^{2} \hat{a}^{\prime \prime}+2 \rho\left(\hat{a}^{\prime}\right)^{3}\right)=-4 \mu \rho\left(x^{+}\right)^{2}\left(\hat{a}^{\prime}\right)^{3}\left(\rho\left(a^{\prime}\right)^{2}\right)^{\prime}, \\
\frac{\partial \mathcal{L}_{T}}{\partial\left(\partial_{-} \partial_{-} a\right)} & =-4 \mu \rho\left(x^{-}\right)^{2}\left(\hat{a}^{\prime}\right)^{3}\left(\rho\left(a^{\prime}\right)^{2}\right)^{\prime}, \\
\frac{\partial \mathcal{L}_{T}}{\partial\left(\partial_{+} \partial_{-} a\right)} & =-8 \mu \rho^{2}\left(\hat{a}^{\prime}\right)^{3}\left(\rho\left(a^{\prime}\right)^{2}\right)^{\prime} .
\end{aligned}
$$

Then from Eqs. (25) and (D2) we have

$$
\left[\rho \hat{a}^{\prime}\right]^{\prime}=\left[\rho \mathcal{H}\left(\rho, \hat{a}^{\prime}\right)\right]^{\prime}-2 \mu\left[\mathcal{K}\left(\rho, \hat{a}^{\prime}, \hat{a}^{\prime \prime}\right)\right]^{\prime}
$$

where

$$
\begin{aligned}
\mathcal{H}\left(\rho, \hat{a}^{\prime}\right) & =2 \epsilon_{2} \rho\left(\hat{a}^{\prime}\right)^{3}-3 \epsilon_{3} \rho^{2}\left(\hat{a}^{\prime}\right)^{5}-8 \mu \rho \hat{a}^{\prime}\left[\left(\hat{a}^{\prime}\right)^{2}+2 \rho \hat{a}^{\prime} \hat{a}^{\prime \prime}\right]^{2}, \\
\mathcal{K}\left(\rho, \hat{a}^{\prime}, \hat{a}^{\prime \prime}\right) & =4 \rho \hat{a}^{\prime}\left[\left(\rho\left(\hat{a}^{\prime}\right)^{2}\right)^{\prime}\right]^{2}+\left[\rho^{2} \hat{a}^{\prime}\left[\rho^{2}\left(\hat{a}^{\prime}\right)^{4}\right]^{\prime}\right]^{\prime}+\rho\left[\rho \hat{a}^{\prime}\left[\rho^{2}\left(\hat{a}^{\prime}\right)^{4}\right]^{\prime}\right]^{\prime} .
\end{aligned}
$$

A logarithmic-type solution is obtained in a perturbative approach (see Ref. [59]) where, to zero order we have

$$
\left[\rho \hat{a}^{\prime}\right]^{\prime}=0 \text {, which leads to } \hat{a}(\rho)=C_{1}+C_{2} \ln (|\rho|)
$$

while the higher order contributions on the right-hand side of Eq. (D3) are properly included by a renormalization procedure that leads to a modification of the argument of the logarithm of the form $\hat{a}=\ln |\rho+g(\rho)|$.

[1] V. B. Berestetskii, E. M. Lifshitz, and L. P. Pitaevskii, Quantum Electrodynamics (Pergamon, New York, 1982).

[2] V.L. Ginzburg, Theoretical Physics and Astrophysics (Pergamon Press, New York, 1979).

[3] W. Heisenberg and H. Euler, Z. Phys. 98, 714 (1936).

[4] R. Karplus and M. Neumann, Phys. Rev. 83, 776 (1951).

[5] J. Schwinger, Phys. Rev. 82, 664 (1951).

[6] J. McKenna and P. M. Platzman, Phys. Rev. 129, 2354 (1963).

[7] T. Erber, Rev. Mod. Phys. 38, 626 (1966).

[8] S. G. Mamaev, V. M. Mostepanenko, and M. I. Eides, Sov. J. Nucl. Phys. 33, 569 (1981).
[9] A. A. Grib, S. G. Mamaev, and V. M. Mostepanenko, Quantum Effects in Intense External Fields (Moscow, Atomizdat, 1980).

[10] R. Battesti, J. Beard, S. Boeser, N. Bruyant, D. Budker et al., Phys. Rep. 765, 1 (2018).

[11] D. Bernard, F. Moulin, F. Amiranoff, A. Braun, J. P. Chambaret, G. Darpentigny, G. Grillon, S. Ranc, and F. Perrone, Eur. Phys. J. D 10, 141 (2000).

[12] C. N. Danson, D. Hillier, N. Hopps, and D. Neely, High Power Laser Sci. Eng. 3, e3 (2015).

[13] C. N. Danson et al., High Power Laser Sci. Eng. 7, e54 (2019). 
[14] T. Heinzl, B. Liesfeld, K.-U. Amthor, H. Schwoerer, R. Sauerbrey, and A. Wipf, Opt. Commun. 267, 318 (2006).

[15] H.-P. Schlenvoigt, T. Heinzl, U. Schramm, T. E. Cowan, and R. Sauerbrey, Phys. Scr. 91, 023010 (2016).

[16] B. King and T. Heintzl, High Power Laser Sci. Eng. 4, e5 (2016).

[17] G. A. Mourou, T. Tajima, and S. V. Bulanov, Rev. Mod. Phys. 78, 309 (2006).

[18] M. Marklund and P. K. Shukla, Rev. Mod. Phys. 78, 591 (2006).

[19] D. Tommasini, A. Ferrando, and M. Seco, Phys. Rev. A 77, 042101 (2008).

[20] A. Paredes, D. Novoa, and D. Tommasini, Phys. Rev. A 90, 063803 (2014).

[21] A. Di Piazza, C. Müller, K. Z. Hatsagortsyan, and C. H. Keitel, Rev. Mod. Phys. 84, 1177 (2012).

[22] R. Battesti and C. Rizzo, Rep. Prog. Phys. 76, 016401 (2013).

[23] J. K. Koga, S. V. Bulanov, T. Z. Esirkepov, A. S. Pirozhkov, M. Kando, and N. N. Rosanov, Phys. Rev. A 86, 053823 (2012).

[24] F. Karbstein and R. Shaisultanov, Phys. Rev. D 91, 113002 (2015).

[25] H. Gies, F. Karbstein, C. Kohlfuerst, and N. Seegert, Phys. Rev. D 97, 076002 (2018).

[26] A. Angioi and A. Di Piazza, Rend. Fis. Acc. Lincei 30, 17 (2019).

[27] S. V. Bulanov, Rend. Fis. Acc. Lincei 30, 5 (2019).

[28] P. Zhang, S. S. Bulanov, D. Seipt, A. V. Arefiev, and A. G. R. Thomas, Phys. Plasmas 27, 050601 (2020).

[29] A. Di Piazza, M. Tamburini, S. Meuren, and C. H. Keitel, Phys. Rev. D 97, 056028 (2018).

[30] F. Karbstein, H. Gies, M. Reuter, and M. Zepf, Phys. Rev. D 92, 071301 (2015).

[31] V. I. Ritus, Sov. Phys. JETP 30, 1181 (1970).

[32] V. I. Ritus, Ann. Phys. (N.Y.) 69, 555 (1972).

[33] N. B. Narozhnyi, Sov. Phys. JETP 28, 371 (1969).

[34] F. Karbstein, H. Gies, M. Reuter, and M. Zepf, Phys. Rev. D 92, 071301 (2015).

[35] S. L. Adler, Ann. Phys. (N.Y.) 67, 599 (1971).

[36] S. V. Bulanov, P. V. Sasorov, F. Pegoraro, H. Kadlecova, S. S. Bulanov, T. Z. Esirkepov, N. N. Rosanov, and G. Korn, Phys. Rev. D 101, 016016 (2020).

[37] A. Di Piazza, M. Tamburini, S. Meuren, and C. H. Keitel, Phys. Rev. A 98, 012134 (2018).

[38] A. Di Piazza, M. Tamburini, S. Meuren, and C. H. Keitel, Phys. Rev. A 99, 022125 (2019).
[39] A. Ilderton, Phys. Rev. D 100, 125018 (2019).

[40] A. Ilderton, B. King, and D. Seipt, Phys. Rev. A 99, 042121 (2019).

[41] F. Sauter, Z. Phys. 69, 742 (1931).

[42] D. J. Korteweg and G. de Vries, Philos. Mag. 39, 422 (1885).

[43] B. B. Kadomstev and V. I. Petviashvili, Sov. Phys. Dokl. 15, 539 (1970).

[44] G. Biondini and D. E. Pelinovsky, Scholarpedia 3, 6539 (2018).

[45] B. Podolsky, Phys. Rev. 62, 68 (1942).

[46] B. Podolsky and C. Kikuchi, Phys. Rev. 65, 228 (1944).

[47] B. Podolsky and P. Schwed, Rev. Mod. Phys. 20, 40 (1948).

[48] A. Barut and G. H. Mullen, Ann. Phys. (N.Y.) 20, 203 (1962)

[49] T. D. Lee and G. C. Wick, Phys. Rev. D 2, 1033 (1970).

[50] R. Turcati and M. J. Neves, Adv. High Energy Phys. 2014, 153953 (2014).

[51] L. H. C. Borges, F. A. Barone, C. A. M. de Melo, and F. E. Barone, Nucl. Phys. B944, 114634 (2019).

[52] M. Soljačić and M. Segev, Phys. Rev. A 62, 043817 (2020).

[53] V. P. Gusynin and I. A. Shovkovy, Can. J. Phys. 74, 282 (1996).

[54] V. P. Gusynin and I. A. Shovkovy, J. Math. Phys. (N.Y.) 40, 5406 (1999).

[55] M. Ostrogradski, Mem. Ac. St. Petersburg 6, 385 (1850).

[56] T.-J. Chen, M. Fasiello, E. A. Lim, and A.J. Tolley, J. Cosmol. Astropart. Phys. 02 (2013) 042.

[57] C. Grosse-Knetter, Phys. Rev. D 49, 6709 (1994).

[58] H. Kadlecova, G. Korn, and S. V. Bulanov, Phys. Rev. D 99 , 036002 (2019).

[59] F. Pegoraro and S. V. Bulanov, Phys. Rev. D 100, 036004 (2019).

[60] F. Pegoraro and S. V. Bulanov, Phys. Lett. A 384, 126064 (2020).

[61] P. K. Shukla, M. Marklund, D. D. Tskhakaya, and B. Eliasson, Phys. Plasmas 11, 3767 (2004).

[62] M. Marklund and J. Lundin, Eur. Phys. J. D 55, 319 (2009).

[63] P. Sasorov, T. Z. Esirkepov, F. Pegoraro, and S. V. Bulanov (to be published).

[64] G. B. Whitham, Linear and Nonlinear Waves (Wiley, New York, 1974).

[65] S. P. Novikov, S. V. Manakov, L. P. Pitaevskii, and V. E. Zakharov, Theory of Solitons: The Inverse Scattering Method (Springer-Verlag, Berlin, 1984).

[66] P. Rosenau and J. M. Hyman, Phys. Rev. Lett. 70, 564 (1993). 